\title{
ON HYPOTHESIS TESTS FOR COVARIANCE MATRICES UNDER MULTIVARIATE NORMALITY
}

\author{
Letícia Pereira Pinto $^{1}$ and Sueli Aparecida Mingoti ${ }^{2 *}$
}

Received June 11, 2012 / Accepted April 06, 2014

\begin{abstract}
In this paper we proposed a new statistical test for testing the covariance matrix in one population under multivariate normal assumption. In general, the proposed and the likelihood-ratio tests resulted in larger values of estimated powers than VMAX for bivariate and trivariate cases. VMAX was not sensitive to general changes in the covariance (correlation) structure. The advantage of the new test is that it is based on the comparison of all elements of the postulated covariance matrix under the null hypothesis with their respective maximum likelihood sample estimates and therefore, it does not restrict the information of the covariance matrix into a scalar number such as the determinant or trace, for example. Due to the fact that it is based on the maximum likelihood estimates and the Fisher information matrix, it can be used for data coming from distribution other than the multivariate normal.
\end{abstract}

Keywords: covariance matrix, hypothesis testing, Fisher information matrix.

\section{INTRODUCTION}

Statistical tests for covariance matrix has been the issue of many papers particularly in quality control for monitoring processes using several quality characteristics simultaneously, or in calibrations studies (Li \& Tsung, 2011). They are also used to verify the assumption of equal covariance matrices when performing statistical tests to compare the vector means of independent populations, such as in marketing research in the comparison of consumers profiles of different segments, among many other applications (Montgomery, 2008).

A well-known statistical test used to verify if the covariance matrix of a population (or process) has a certain specified structure is the generalized variance, $|\boldsymbol{S}|$, which is based on the determinant of the sample covariance matrix (see Alt, 1985; Djauhari, 2005). Tests based on transformations of $|\boldsymbol{S}|$ are also found in the literature (García-Diaz, 2007). Although the idea of summarizing the information of the covariance matrix into a scalar number is appealing, the fact that different

\footnotetext{
*Corresponding author.

${ }^{1}$ Departament of Computer Science, UFMG, Belo Horizonte, Minas Gerais, Brazil. E-mail: leticiap@dcc.ufmg.br

2 Departament of Statistics, UFMG, Belo Horizonte, Minas Gerais, Brazil. E-mail: sueliam@ est.ufmg.br
} 
matrices can have equal or similar determinants makes the generalized variance to fail in detecting some types of changes in the covariance matrix when they take place. The assumption of a normal distribution as an approximated distribution for $|\boldsymbol{S}|$, may also restrict its use in certain areas since it just holds for large sample sizes (Djauhari, 2009). In quality control, for example, it is common to use samples of sizes $n=4$ or 5, cases for which the normal approximation would not be valid. The exact distribution of $|\boldsymbol{S}|$ for the bivariate case is known and it is related to a chi-square distribution. However, for $p>2$ variables the exact distribution of $|S|$ does not belong to any class of known probability distributions (Anderson, 1958; Aparisi et al., 1999).

The likelihood-ratio test was presented by Anderson (1958), in a general context for multivariate normal populations and by Alt (1985), as a tool to monitor the covariance matrix in quality control. However, the test was based on the chi-square distribution for the test statistic which is valid asymptotically, only. Costa \& Machado (2009; 2008), proposed the VMAX based on the maximum of the $p$ standardized sample variances, $p$ being the number of variables or quality characteristics. VMAX was more efficient than the generalized variance to monitor processes presenting better capability at detecting variances shifts. The critical region of the test (or control chart) was obtained by numerical integration which involves non-central chi-square distributions. More recently, Quinino et al. (2012), proposed the VMIX as a tool to monitor the variability structure of two quality characteristics under the assumption of bivariate normal distribution. In their paper it was shown that VMIX was more efficient than the generalized variance control chart and better than VMAX, for some cases.

A more general method was proposed by Sullivan et al. (2007), considering the vector of parameters $\theta$ which includes the elements of the mean vector and the covariance matrix. The estimated vector of $\boldsymbol{\theta}$ is compared with the vector $\boldsymbol{\theta}$ specified in the null hypothesis by means of a test statistic which has an approximated chi-square distribution under the null hypothesis. What is interesting in Sullivan et al. (2007) test is that there is no restriction on the structure of the covariance matrix postulated on the alternative hypothesis and it can be used for normal and non-normal distributions being capable of testing the whole vector of parameters as well as parts of it. As an illustration for the bivariate case, one could be interested in testing the vector $\boldsymbol{\theta}$ containing all 5 parameters (two means, two variances and one covariance); the parameter vector $\theta$ containing the two means or two variances only, or containing just the variances and the covariances, among other possibilities. Other statistical tests for covariance matrices can be found in the literature (see Yeh et al., 2006; 2012, among others).

In this paper we discuss the performance of the Sullivan et al. (2007), when used to test the covariance matrix. Its performance will be compared to the likelihood-ratio and the VMAX tests by using the exact distribution of the test statistic, as well as the chi-square distribution as an approximation. This paper is organized as follows: in Section 2 the statistical tests are presented; in Section 3 the results of the comparisons of the tests are shown followed by numerical examples in Section 4 and final remarks in Section 5. 


\section{STATISTICAL TESTS FOR COVARIANCE MATRICES}

For all tests presented it this section, it is assumed that $X_{1}, X_{2}, \ldots, X_{n}$, where $\mathbf{X}_{\mathbf{k}}=$ $\left(X_{k 1}, X_{k 2}, \ldots, X_{k p}\right)^{\prime}, k=1,2, \ldots, n$, is a random sample of size $n$ from a $p$-variate normal distribution with the mean vector $\boldsymbol{\mu}=\left(\mu_{1}, \mu_{2}, \ldots, \mu_{p}\right)^{\prime}$ and covariance matrix $\boldsymbol{\Sigma}$, a $p \times p$ positive definite matrix, where $p$ is the number of random variables.

\subsection{The likelihood-ratio test}

The likelihood-ratio is a well-known method of hypothesis testing (Alt, 1985; Casella \& Berger, 2002), and it can be used to construct a test for the covariance matrix as follows. Under the $p$-variate normal distribution assumption, the likelihood function is given as

$$
L\left(\boldsymbol{x}_{\mathbf{1}}, \boldsymbol{x}_{\mathbf{2}}, \ldots, \boldsymbol{x}_{\boldsymbol{n}}\right)=\frac{1}{(2 \pi)^{n p / 2}|\boldsymbol{\Sigma}|^{n / 2}} \prod_{k=1}^{n} \exp \left[-\frac{1}{2}\left(\boldsymbol{x}_{k}-\boldsymbol{\mu}\right)^{\prime} \boldsymbol{\Sigma}^{-1}\left(\boldsymbol{x}_{\boldsymbol{k}}-\boldsymbol{\mu}\right)\right]
$$

where $\boldsymbol{x}_{\boldsymbol{k}}=\left(x_{k 1}, x_{k 2}, \ldots, x_{k p}\right)^{\prime}$ is the observed vector for the $k t h$ sample element, $k=$ $1,2, \ldots, n$, and $|$.$| denotes the determinant of the matrix. Let the null and the alternative hy-$ pothesis be defined as $H_{0}: \mathbf{\Sigma}=\boldsymbol{\Sigma}_{\mathbf{0}}, H_{1}: \mathbf{\Sigma} \neq \boldsymbol{\Sigma}_{\mathbf{0}}, \boldsymbol{\mu}$ unknown. Under the null hypothesis the maximum $\left(L_{o}\right)$ of the likelihood function (1) is achieved when the vector $\boldsymbol{\mu}$ is taken as the sample mean vector $\overline{\boldsymbol{x}}$ and $\boldsymbol{\Sigma}=\boldsymbol{\Sigma}_{\mathbf{0}}$. Under the whole parametric space the maximum $\left(L_{1}\right)$ is achieved when the vector $\boldsymbol{\mu}$ is taken as the sample mean vector $\overline{\boldsymbol{x}}$ and the covariance matrix is estimated as $\hat{\Sigma}$, where

$$
\overline{\boldsymbol{x}}=\frac{1}{n} \sum_{k=1}^{n} \boldsymbol{x}_{\boldsymbol{k}} ; \quad \hat{\boldsymbol{\Sigma}}_{p \times p}=\frac{(n-1)}{n} \boldsymbol{S}_{p \times p}
$$

$S_{p \times p}=(n-1)^{-1} \sum_{k=1}^{n}\left(x_{k}-\bar{x}\right)\left(x_{k}-\bar{x}\right)^{\prime}$ is the sample covariance matrix. The likelihood-ratio test statistic is then given by

$$
W=-2 \ln \left(L_{0} / L_{1}\right)=-p n+p n \ln (n)-n \ln \left(\left|\boldsymbol{A} \Sigma_{\mathbf{0}}^{-1}\right|\right)+\operatorname{tr}\left(\boldsymbol{\Sigma}_{\mathbf{0}}^{-1} \boldsymbol{A}\right)
$$

where $\boldsymbol{A}=(n-1) \boldsymbol{S}$ and $\operatorname{tr}($.$) is the trace operator. The distribution of W$ under the null hypothesis is asymptotically chi-square and $H_{0}$ is rejected for values of $W$ larger than the constant $L S C=\chi_{\alpha, p(p+1) / 2}^{2}$, which is a value taken from a chi-square distribution with $p(p+1) / 2 \mathrm{de}-$ grees of freedom, whose area above is equal to the significance level of the test, $\alpha, 0<\alpha<1$. In the quality control terminology $\alpha$ is the false alarms rate. When the vector mean $\boldsymbol{\mu}$ is known under the null hypothesis, the test statistic in (3) reduces to

$$
W=-2 \ln \left(L_{0} / L_{1}\right)=-p n-n \ln \left(\left|\boldsymbol{\Sigma}_{\mathbf{0}}^{-1} \hat{\Sigma}\right|\right)+n t r\left(\boldsymbol{\Sigma}_{\mathbf{0}}^{-1} \hat{\Sigma}\right)
$$

where $\hat{\mathbf{\Sigma}}=(n)^{-1} \sum_{k=1}^{n}\left(x_{k}-\mu\right)\left(x_{k}-\mu\right)^{\prime}$.

\subsection{The VMAX test}

Costa \& Machado (2008), proposed a new control chart to monitor the covariance matrix $\mathbf{\Sigma}$ for bivariate normal populations when the mean vector $\boldsymbol{\mu}=\left(\mu_{1}, \mu_{2}\right)^{\prime}$, was known. Let $\boldsymbol{X}=$ 
$\left(X_{1}, X_{2}\right)^{\prime}$ be the random vector of interest and $H_{0}: \Sigma=\Sigma_{\mathbf{0}}, H_{1}: \Sigma=\Sigma_{\mathbf{1}}$, be the null and the alternative hypothesis, where

$$
\mathbf{\Sigma}_{\mathbf{0}}=\left[\begin{array}{cc}
\sigma_{1}^{2} & \sigma_{12} \\
\sigma_{21} & \sigma_{2}^{2}
\end{array}\right] \quad \text { and } \quad \Sigma_{\mathbf{1}}=\left[\begin{array}{cc}
a^{2} \sigma_{1}^{2} & a b \sigma_{12} \\
a b \sigma_{21} & b^{2} \sigma_{2}^{2}
\end{array}\right]
$$

$a>0, b>0$ are constants, $\sigma_{1}^{2}, \sigma_{2}^{2}$ and $\sigma_{12}=\sigma_{21}$ are the variances and the covariances of $X_{1}$ and $X_{2}$, respectively, under the null hypothesis; $a^{2} \sigma_{1}^{2}, b^{2} \sigma_{2}^{2}$ and $a b \sigma_{12}$, are the variances and the covariances of $X_{1}$ and $X_{2}$, respectively, under the alternative hypothesis. Under this structure, changes in the variances and covariances values of $X_{1}$ and $X_{2}$ are allowed but the correlation structure is not affected by the changes.

The VMAX test statistic is defined as

$$
V M A X=\max \left\{S_{1}^{2}, S_{2}^{2}\right\}
$$

where

$$
S_{1}^{2}=\frac{\sum_{k=1}^{n}\left(x_{k 1}-\mu_{1}\right)^{2}}{n \sigma_{1}^{2}} ; \quad S_{2}^{2}=\frac{\sum_{k=1}^{n}\left(x_{k 2}-\mu_{2}\right)^{2}}{n \sigma_{2}^{2}},
$$

$\left(x_{k 1}, x_{k 2}\right), k=1,2, \ldots, n$, are the sample values of the random vector $\boldsymbol{X}=\left(X_{1}, X_{2}\right)^{\prime}$. For a given significance level $\alpha, 0<\alpha<1$, the critical region of the test is obtained by solving the equation (6) for $C L$, considering $a=b=1$. The null hypothesis is then rejected for any value of (5) larger than the critical constant $C L$. In the quality control field the constant $C L$ is the control limit.

$$
\alpha=1-\int_{0}^{n C L / a^{2}} \operatorname{Pr}\left[\chi_{n,\left(t \rho^{2} / 1-\rho^{2}\right)}^{2}<\frac{n C L}{b^{2}\left(1-\rho^{2}\right)}\right] \frac{e^{-t / 2}}{2^{n / 2} \Gamma\left(\frac{n}{2}\right)} t^{(n / 2)-1} d t
$$

where $\chi_{n,\left(t \rho^{2} / 1-\rho^{2}\right)}^{2}$ represents a chi-square distribution with $n$ degrees of freedom and noncentrality parameter given by $t \rho^{2} /\left(1-\rho^{2}\right), \Gamma($.$) is the Gamma function, \rho$ is the correlation coefficient between $X_{1}$ and $X_{2}$ and $\operatorname{Pr}[$.] denotes the probability of the considered event. Under the alternative hypothesis the expression in (5) is used to compute the power of VMAX test. When the means $\left(\mu_{1}, \mu_{2}\right)$ are unknown, they can be replaced by the respective sample means.

Details about the equation (6) can be found in the Appendix of Costa \& Machado's (2008; 2009) papers. Basically, it was derived taking into consideration the fact that under the bivariate normal distribution assumption, $n S_{1}^{2} / a^{2}$ has a chi-square distribution with $n$ degrees of freedom $\left(\chi_{n}^{2}\right)$. It can also be shown that conditioned on $\boldsymbol{x}_{k}=\left(x_{k 1}, x_{k 2}, \ldots, x_{k p}\right)^{\prime}$, the random variable defined as

$$
\frac{n S_{2}^{2}}{b^{2}\left(1-\rho^{2}\right)}
$$

has a non-central chi-square distribution with $n$ degrees of freedom and non-centrality parameter given as $\left(\rho^{2} / 1-\rho^{2}\right) \chi_{n}^{2}$. The equation (6) follows taking into account that

$$
p_{w}=1-\operatorname{Pr}\left[\left(S_{1}^{2} \leq C L\right) \cap\left(S_{2}^{2} \leq C L\right)\right]
$$

where $p_{w}=\alpha$ when $a=b=1$ (under the null hypothesis), and $p_{w}$ is the power of the test under the alternative hypothesis. 
Costa \& Machado (2008), also considered the case where the covariance matrix $\boldsymbol{\Sigma}_{1}$ was defined as

$$
\boldsymbol{\Sigma}_{\mathbf{1}}=\left[\begin{array}{cc}
a^{2} \sigma_{1}^{2} & \sigma_{12} \\
\sigma_{21} & b^{2} \sigma_{2}^{2}
\end{array}\right] .
$$

In this case, under the alternative hypothesis the correlation coefficient between $X_{1}$ and $X_{2}$ would change to $\rho=\sigma_{i j} / a b \sigma_{1} \sigma_{2}$, where $\sigma_{1}, \sigma_{2}$, are the standard deviations of the variables $X_{1}$ and $X_{2}$, respectively.

VMAX test was extended by Costa \& Machado (2009), for the multivariate case $(p>2)$ by using a similar approach as taken to the bivariate normal population. Numerical integration was used to obtain the critical constant $C L$ of the test.

\subsection{Sullivan et al. (2007) statistical test}

Sullivan et al. (2007), proposed a statistical test that can be applied for two groups of consecutive independent homogeneous observations with the purpose of comparing the parameters of the distributions of both groups. This test is of importance in quality control since it can be used to compare the process parameters before and after some identified change point in the structure of the sample observations. In a more general context Sullivan et al. (2007) test could be applied in the comparison of the vector means and/or the covariance matrices of two independent groups.

Let's suppose that the parameters of the distributions before $(A)$ and after $(B)$ the change point, are described by the parameter vector $\boldsymbol{\theta}$ which consists of the elements of the vector mean $\boldsymbol{\mu}$ and the covariance matrix $\boldsymbol{\Sigma}$. As an illustration, for the bivariate normal distribution the vector $\boldsymbol{\theta}$ can be defined as $\boldsymbol{\theta}=\left(\mu_{1}, \mu_{2}, \sigma_{1}^{2}, \sigma_{12}, \sigma_{2}^{2}\right)^{\prime}$ or $\boldsymbol{\theta}=\left(\mu_{1}, \mu_{2}, \sigma_{1}, \rho_{12}, \sigma_{2}\right)^{\prime}$, where $\sigma_{12}$ and $\rho_{12}$ are the covariance and correlation between the two variables and $\sigma_{i}$ is the standard deviation of the variable $X_{i}, i=1,2$. Let $\boldsymbol{\theta}_{A}$ and $\boldsymbol{\theta}_{B}$ be the parameter vectors of the groups of observations $A$ and $B$ respectively, and $\hat{\boldsymbol{\theta}}_{A}$ and $\hat{\boldsymbol{\theta}}_{B}$ be their respective maximum likelihood estimators. Let the $\left(k_{0} \times 1\right)$ vector $\hat{\boldsymbol{\delta}}=\left(\hat{\boldsymbol{\theta}}_{A}-\hat{\boldsymbol{\theta}}_{B}\right)$, be the maximum likelihood estimator of the difference vector $\boldsymbol{\delta}=\left(\boldsymbol{\theta}_{\boldsymbol{A}}-\boldsymbol{\theta}_{\boldsymbol{B}}\right)$, where $k_{0}$ is the number of parameters being compared. As well known, under general regularity conditions, the maximum likelihood estimators have asymptotically a multivariate normal distribution (Casella \& Berger, 2002). Therefore, under the null hypothesis, asymptotically, the quadratic form

$$
\chi_{A, B}^{2}=\hat{\boldsymbol{\delta}}^{\prime} \hat{\boldsymbol{\Sigma}}_{\hat{\boldsymbol{\delta}}}^{-\mathbf{1}} \hat{\boldsymbol{\delta}}
$$

has a chi-square distribution with $k_{0}$ degrees of freedom, where $\boldsymbol{\Sigma}_{\delta}$ and $\boldsymbol{\Sigma}_{\hat{\delta}}$ are the theoretical and the estimated covariance matrices of $\hat{\delta}$, respectively. The null hypothesis corresponds to the non-changing in the vector of parameters, i.e., $\boldsymbol{\delta}=\mathbf{0}$. The covariance matrix $\boldsymbol{\Sigma}_{\hat{\delta}}$ is approximated by the Fisher information matrix (Casella \& Berger, 2002), and it is estimated by using an algebraic expression for the expected value of the Hessian matrix, which is then evaluated at the maximum likelihood estimates of the parameters.

The framework used in Sullivan et al. (2007) test makes it possible to build a test for the covariance matrix components as shown next. 


\subsection{An Adaptation of Sullivan et al. (2007) to test the covariance matrix}

In this section we will describe an adaptation of the Sullivan et al. (2007) test for the situation in which only the covariance matrix of the population is concerned. From all over this paper we will call this test as an adapted Sullivan et al.

Let $p=2, \mu_{1}$ and $\mu_{2}$ pre-specified and $\boldsymbol{\theta}=\left(\sigma_{1}, \rho_{12}, \sigma_{2}\right)^{\prime}$ be the parameter vector from a bivariate normal distribution. Let $\hat{\boldsymbol{\theta}}$ be the vector containing the maximum likelihood estimates of the parameters in $\boldsymbol{\theta}$. Let $H_{0}: \boldsymbol{\theta}=\boldsymbol{\theta}_{\mathbf{0}}$ and $H_{1}: \boldsymbol{\theta}=\boldsymbol{\theta}_{\mathbf{1}}$, be the null and the alternative hypothesis which are equivalent to $H_{0}: \delta=\mathbf{0}$ and $H_{0}: \delta \neq \mathbf{0}$, where $\delta=\boldsymbol{\theta}-\boldsymbol{\theta}_{\mathbf{0}}$. Let the test statistic be defined as

$$
\chi_{s}^{2}=\hat{\delta}^{\prime} \Sigma_{\hat{\delta}}^{-1} \hat{\delta}
$$

where $\hat{\boldsymbol{\delta}}=\hat{\boldsymbol{\theta}}-\boldsymbol{\theta}_{\mathbf{0}}$. Under $H_{0}$, asymptotically, the test statistic in (7) has a chi-square distribution with $k_{0}=3$ degrees of freedom being the null hypothesis rejected for large values of (7), according to the significance level of the test. Under the bivariate normality assumption the covariance matrix $\Sigma_{\hat{\delta}}$ is obtained through the Fisher information matrix (see Beyer, 1978; Sullivan et al., 2007), which is given by

$$
\Sigma_{\hat{\delta}}=\left[\begin{array}{rrr}
d & -e & f \\
-e & h & -g \\
f & -g & i
\end{array}\right]
$$

where

$$
\begin{gathered}
d=\frac{\sigma_{1}^{2}}{2 n}, \quad e=\frac{\rho_{12}\left(\rho_{12}^{2}-1\right) \sigma_{1}}{2 n}, \quad f=\frac{\rho_{12}^{2} \sigma_{1} \sigma_{2}}{2 n}, \\
g=\frac{\rho_{12}\left(\rho_{12}^{2}-1\right) \sigma_{2}}{2 n}, h=\frac{\left(\rho_{12}^{2}-1\right)}{n} \text { and } i=\frac{\sigma_{2}^{2}}{2 n},
\end{gathered}
$$

being the values of $\sigma_{1}, \sigma_{2}, \rho_{12}, \sigma_{1}^{2}, \sigma_{2}^{2}, \sigma_{12}$ as specified in $H_{0}$, where $\sigma_{1}^{2}, \sigma_{2}^{2}$ are the variances of $X_{1}$ and $X_{2}$ variables, respectively.

The adapted Sullivan et al. test for the covariance matrix can also be extended for $p>2$ variables. Let $\boldsymbol{X}$ be a $(p \times 1)$ random vector with multivariate normal distribution, with mean vector $\boldsymbol{\mu}(\boldsymbol{\theta})$ and covariance matrix $\boldsymbol{\Sigma}(\boldsymbol{\theta})$, where $\boldsymbol{\mu}(\boldsymbol{\theta})=\left(\mu_{1}(\theta), \mu_{2}(\theta), \ldots, \mu_{p}(\theta)\right)^{\prime}$. In this case the typical $(i, j)$ element of the Fisher information matrix is given by

$$
I_{i, j}=\frac{\partial \mu}{\partial \theta_{i}} \Sigma^{-1} \frac{\partial \mu^{\prime}}{\partial \theta_{j}}+\frac{1}{2} \operatorname{tr}\left[\Sigma^{-1} \frac{\partial \Sigma}{\partial \theta_{i}} \Sigma^{-1} \frac{\partial \Sigma}{\partial \theta_{j}}\right]
$$

where

$$
\frac{\partial \boldsymbol{\mu}}{\partial \theta_{i}}=\left[\frac{\partial \mu_{1}}{\partial \theta_{i}}, \frac{\partial \mu_{2}}{\partial \theta_{i}}, \ldots, \frac{\partial \mu_{p}}{\partial \theta_{i}}\right] ; \frac{\partial \boldsymbol{\Sigma}}{\partial \theta_{i}}=\left[\begin{array}{cccc}
\frac{\partial \Sigma_{1,1}}{\partial \theta_{i}} & \frac{\partial \Sigma_{1,2}}{\partial \theta_{i}} & \ldots & \frac{\partial \Sigma_{1, p}}{\partial \theta_{i}} \\
\frac{\partial \Sigma_{2,1}}{\partial \theta_{i}} & \frac{\partial \Sigma_{2,2}}{\partial \theta_{i}} & \ldots & \frac{\partial \Sigma_{2, p}}{\partial \theta_{i}} \\
\vdots & \vdots & \vdots & \vdots \\
\frac{\partial \Sigma_{p, 1}}{\partial \theta_{i}} & \frac{\partial \Sigma_{p, 2}}{\partial \theta_{i}} & \cdots & \frac{\partial \Sigma_{p, p}}{\partial \theta_{i}}
\end{array}\right]
$$


$\operatorname{tr}($.$) is the trace operator and \Sigma_{i, j}$ is the $(i, j)$ element of the covariance matrix $\boldsymbol{\Sigma}$.

A possible advantage of the adapted Sullivan et al. test regarding to the VMAX and the likelihood-ratio tests comes from the fact that all the parameters of the covariance matrix postulated in the null hypothesis are compared in its test statistic, one by one, with their respective maximum likelihood estimates, so that there is no reduction of the covariance matrix information into a single measure such as the determinant, or others. It also allows the correlation coefficients under the null and the alternative hypothesis to be completely different being therefore, a more general test. The test can also be used for non-normal multivariate data since it is based on the maximum likelihood estimates and the Fisher information matrix. It also can be used to test shifts in the variances of the variables only.

\section{COMPARISON OF THE STATISTICAL TESTS}

The power of the adapted Sullivan et al., the VMAX and the likelihood-ratio tests were estimated by using Monte Carlo simulation under the multivariate normal distribution assumption. The null hypothesis is $H_{0}: \boldsymbol{\Sigma}=\boldsymbol{\Sigma}_{\mathbf{0}}$, where $\boldsymbol{\Sigma}_{\mathbf{0}}$ is pre-specified. For the adapted Sullivan et al. test as well as for the likelihood-ratio, the exact and the asymptotic chi-square distributions of the test statistics were also consider to determine the respective rejection regions of $H_{0}$.

In this paper, in the implementation of the VMAX test for the bivariate normal distribution, the Gauss-Legendre and the Pegasus method were used to sort out the numeric integration and to determine the zeros of the function given in (6) in Section 2.2 (see Davis \& Rabinowitz, 1984, for details). Due to the fact that the values of $C L$ for $p=2$ obtained by using numeric integration were similar to those obtained by using simulation, the value of $C L$ for $p=3$ was obtained by simulation only.

In order to determine the critical region of the tests by the exact distribution (except for VMAX, $p=2), M=50.000$ random samples of fixed sizes $n=5,10,25,50$ and 100 were generated from a $p$-variate normal distribution with mean vector $\boldsymbol{\mu}$ and covariance matrix $\boldsymbol{\Sigma}_{\mathbf{0}}$ as stipulated in the null hypothesis. For each sample the respective test statistic was calculated and the empirical distribution was built based on the $M$ obtained sample values. By using this distribution the critical region of the test was determined according to the pre-specified significance level $\alpha=$ 0.05 for the tests. After the determination of the critical region, the respective type I error and power of the tests were estimated as follows. First, 10.000 random samples were generated from a multivariate normal distribution under the null hypothesis considering $\boldsymbol{\Sigma}=\boldsymbol{\Sigma}_{\mathbf{0}}$. For each sample and each test, the null hypothesis was tested and the proportion of rejection was calculated given an estimate of type I error of the test. Afterwards, 10.000 samples were generated from a multivariate normal distribution under the alternative hypothesis, $H_{1}: \Sigma=\Sigma_{\mathbf{1}}$, considering different $\boldsymbol{\Sigma}_{\mathbf{1}}$ settings, $\boldsymbol{\Sigma}_{\mathbf{1}} \neq \boldsymbol{\Sigma}_{\mathbf{0}}$. Again for each sample and each test, the null hypothesis was tested and the proportion of rejection was computed given an estimate of the power of the test. This procedure was repeated $k=50$ times under the null and the alternative hypothesis. At the end, average estimates of the type I error and the power were obtained by taking the average over 
all 50 repetitions for each test, respectively. Without loss of generality this study was performed considering one particular structure for the matrix $\boldsymbol{\Sigma}_{\mathbf{0}}$ for $p=2,3$ and assuming $\boldsymbol{\mu}=\mathbf{0}$ under $H_{0}$ and $H_{1}$.

The simulated models under the alternative hypothesis (see Tables 1 and 2), were chosen to make it possible to evaluate whether the traditional likelihood-ratio, the adapted Sullivan et al. and the VMAX tests were able to detect small and large differences from $\boldsymbol{\Sigma}_{\mathbf{0}}$. In models 1-6 for $p=2$, and models $1,6,7$ for $p=3$, changes in the variances and in the correlation structure were allowed. Models 7-10 for $p=2,4$ and 8 for $p=3$, were more restrict since the variances were allowed to change but the correlation (in model 4) and the covariance (in model 10) structures remained the same as under the null hypothesis. On the contrary, the models 2,3 and 5 for $p=3$, have the same variances as $H_{0}$ but different correlation and covariance structures.

Tables 1 and 2 also present the ratio of the covariance matrices traces and determinants under $H_{0}$ and $H_{1}$ as well as the ratio of the maximum of the variances under the alternative and null simulated models. The ratio of the determinants for the bivariate models ranged from 1 (cases 2 and 3) to 2.67 (case 5), which corresponds to an increase of 0 to $166,67 \%$ in the determinant under $H_{1}$ compared to the determinant under $H_{0}$. For the trivariate models the ratio ranged from 1.13 to 9.73 (case 8 ).

\subsection{Comparison by using the exact distribution of the tests statistics}

The average proportions of rejection of $H_{0}$ for each test discussed in this paper are shown in Tables 3 and 4 , for $p=2$, under the null and the alternative hypothesis models. When the exact distribution was used to build the critical region of the tests, the estimates of the type I error were 0.05 as expected. Considering only the exact distributions of the test statistics, the adapted Sullivan et al. test (SE) performed better than the likelihood-ratio (LRE) for all sample sizes except in cases 2, 4 and 5, for $n=50$ and 100, cases which both tests were similar. Compared to VMAX the SE test presented larger power values in cases 1-6 for all sample sizes. Both tests had similar performance for cases 7-10 with some advantage to VMAX for smaller sample sizes.

It is important to point out the outstanding superiority of the SE test in case 2 when compared to VMAX even for smaller samples sizes (SE average power $\geq 0.58$; VMAX average power around 0.05 for all samples sizes). This is an important point in favour of SE since the correlation structure of $\boldsymbol{\Sigma}_{\mathbf{1}}$ and $\boldsymbol{\Sigma}_{\mathbf{0}}$ are completely different (opposite covariance structure), although the variances of the two random variables are the same as well as the generalized variance. VMAX presented also poor performance for situations where the matrix $\boldsymbol{\Sigma}_{\mathbf{1}}$ was diagonal with variances similar to the matrix $\boldsymbol{\Sigma}_{\mathbf{0}}$ (cases 1, 3 and 4). In these cases, the maximum power values were achieved at $n=100$ being equal to 0.05 (case 1), 0.18 (case 3) and 0.41 (case 4), values much lower than the obtained results from SE and LRE. Just as an example, for $n=25$ the power values from SE were above $0.7(0.77 ; 0.79 ; 0.82)$ and above 0.6 from LRE $(0.63 ; 0.67 ; 0.72)$. Even for case 5 which the increase of the generalized variance under the alternative hypothesis was $166,67 \%$ compared to the null hypothesis, the VMAX did not performed well for samples 
Table 1 - Simulated models $-p=2$ variables.

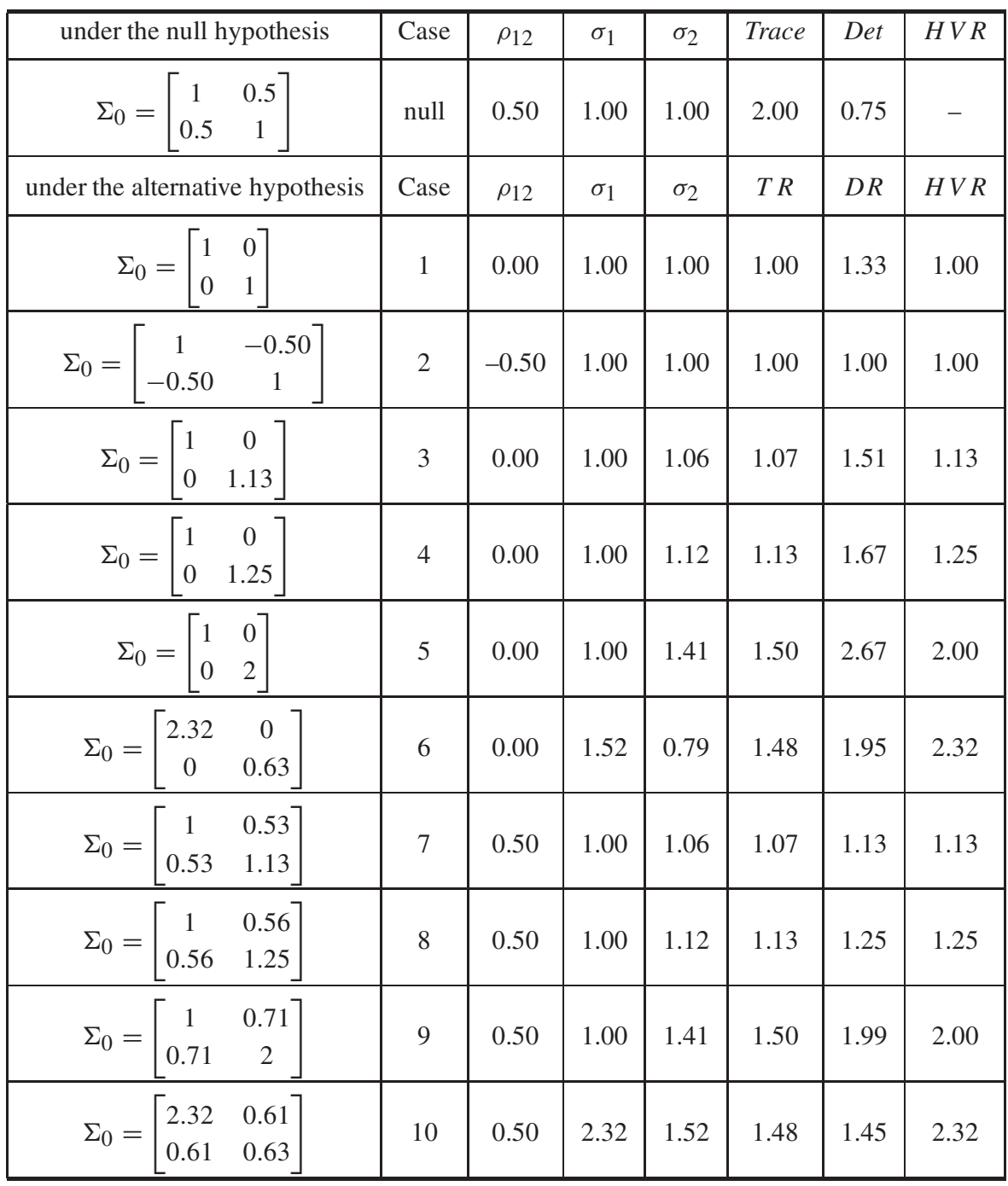

(*) Det: determinant; $T R$ and $D R$ : ratios between the traces and the determinants of the covariance matrices under $H_{1}$ and $H_{0}$, respectively; $H V R$ : ratio between the maximum of the variances under $H_{1}$ and $H_{0} . \rho_{12}$ is the correlation between both variables. $\sigma_{i}$ is the standard deviation of variable $X_{1}, i=1,2$.

of size $n=5$ (power $=0.29$ ) reaching a power equal 0.74 for $n=25$ (for this particular value of $n$, the power values of SE and LRE were 0.96 and 0.93 , respectively). That is not very surprising since the test statistic of VMAX is based only upon the maximum ratio between the sample and the population variances postulated in the null hypothesis.

When the matrix $\Sigma_{\mathbf{1}}$ is diagonal with variances larger than $\boldsymbol{\Sigma}_{\mathbf{0}}$ (cases 5 and 6 ), the power of VMAX increases for all sample sizes but the values are still lower than SE and LRE, except 
Table 2 - Simulated models $-p=3$ variables.

\begin{tabular}{|c|c|c|c|c|c|c|c|c|c|c|c|}
\hline \multicolumn{4}{|c|}{ under the null hypothesis } & Case & $\rho_{12}$ & $\rho_{13}$ & $\rho_{23}$ & Det & Trace & $D R$ & $H V R$ \\
\hline$\Sigma_{1}=$ & {$\left[\begin{array}{c}1 \\
0.6 \\
0.6\end{array}\right.$} & $\begin{array}{c}0.6 \\
1 \\
0.8\end{array}$ & $\left.\begin{array}{c}0.6 \\
0.8 \\
1\end{array}\right]$ & null & 0.60 & 0.60 & 0.80 & 0.22 & 3.00 & - & - \\
\hline under the & alterna & ative $h$ & hypothesis & Case & $\rho_{12}$ & $\rho_{13}$ & $\rho_{23}$ & Det & Trace & $D R$ & $H V R$ \\
\hline$\Sigma_{1}=$ & {$\left[\begin{array}{l}2 \\
0 \\
0\end{array}\right.$} & $\begin{array}{c}0 \\
0.5 \\
0\end{array}$ & $\left.\begin{array}{c}0 \\
0 \\
0.25\end{array}\right]$ & 1 & 0.00 & 0.00 & 0.00 & 0.25 & 0.92 & 1.13 & 2.00 \\
\hline$\Sigma_{1}=$ & {$\left[\begin{array}{c}1 \\
0.3 \\
0.2\end{array}\right.$} & $\begin{array}{c}0.3 \\
1 \\
0.8\end{array}$ & $\left.\begin{array}{c}0.2 \\
0.8 \\
1\end{array}\right]$ & 2 & 0.30 & 0.20 & 0.80 & 0.33 & 1.00 & 1.50 & 1.00 \\
\hline$\Sigma_{1}=$ & $=\left[\begin{array}{l}1 \\
0 \\
0\end{array}\right.$ & $\begin{array}{c}0 \\
1 \\
0.8\end{array}$ & $\left.\begin{array}{c}0 \\
0.8 \\
1\end{array}\right]$ & 3 & 0.00 & 0.00 & 0.80 & 0.36 & 1.00 & 1.64 & 1.00 \\
\hline$\Sigma_{1}=$ & $\begin{array}{c}1.2 \\
0.72 \\
0.72\end{array}$ & $\begin{array}{c}0.72 \\
1.2 \\
0.96\end{array}$ & $\left.\begin{array}{c}0.72 \\
0.96 \\
1.2\end{array}\right]$ & 4 & 0.60 & 0.60 & 0.80 & 0.37 & 1.20 & 1.68 & 1.20 \\
\hline$\Sigma_{1}=$ & {$\left[\begin{array}{c}1 \\
0.3 \\
0.3\end{array}\right.$} & $\begin{array}{c}0.3 \\
1 \\
0.4\end{array}$ & $\left.\begin{array}{c}0.3 \\
0.4 \\
1\end{array}\right]$ & 5 & 0.30 & 0.30 & 0.40 & 0.73 & 1.00 & 3.32 & 1.00 \\
\hline$\Sigma_{1}=$ & $\begin{array}{c}1.2 \\
0.36 \\
0.36\end{array}$ & $\begin{array}{c}0.36 \\
1.2 \\
0.48\end{array}$ & $\left.\begin{array}{c}0.36 \\
0.48 \\
1.2\end{array}\right]$ & 6 & 0.30 & 0.30 & 0.40 & 1.26 & 1.20 & 5.73 & 1.20 \\
\hline$\Sigma_{1}=$ & $\begin{array}{c}1.2 \\
0.36 \\
0.36\end{array}$ & $\begin{array}{c}0.36 \\
1.5 \\
0.48\end{array}$ & $\left.\begin{array}{c}0.36 \\
0.48 \\
1\end{array}\right]$ & 7 & 0.27 & 0.33 & 0.39 & 1.32 & 1.23 & 6.00 & 1.50 \\
\hline$\Sigma_{1}=$ & {$\left[\begin{array}{c}1 \\
0.6 \\
0.6\end{array}\right.$} & $\begin{array}{c}0.6 \\
4 \\
0.8\end{array}$ & $\left.\begin{array}{c}0.6 \\
0.8 \\
1\end{array}\right]$ & 8 & 0.30 & 0.60 & 0.40 & 2.14 & 2.00 & 9.73 & 4.00 \\
\hline
\end{tabular}

(*) Det: determinant; $T R$ and $D R$ : ratios between the traces and the determinants of the covariance matrices under $H_{1}$ and $H_{0}$, respectively; $H V R$ : ratio between the maximum of the variances under $H_{1}$ and $H_{0} . \rho_{i j}$ is the correlation between variables. $X_{i}$ and $X_{j}, i \neq j$.

for $n=50$ and 100, where they all achieved similar power (around 1). For the models 7-10 which the correlation structures are the same under the null and the alternative hypothesis, the performance of the likelihood-ratio test decreased and its power estimates were always lower than SE and VMAX, except in cases 9 and 10 for $n \geq 50$, which all tests presented similar power 
Table 3 - Type I error and power estimates of the tests $-p=2, n=5,10$ and 25.

\begin{tabular}{|c|c|c|c|c|c|c|c|c|c|c|c|c|}
\hline \multicolumn{10}{|c|}{ Average proportion of rejection of the null hypothesis } \\
\hline & \multicolumn{3}{|c|}{$n=5=10$} & \multicolumn{3}{c|}{$n=10$} & \multicolumn{3}{|c|}{$n=25$} \\
\hline Case & LRE & SE & VMAX & LRE & SE & VMAX & LR & LRE & SC & SE & VMAX \\
\hline null & 0.05 & 0.05 & 0.05 & 0.05 & 0.05 & 0.05 & 0.07 & 0.05 & 0.06 & 0.05 & 0.05 \\
1 & 0.10 & 0.25 & 0.05 & 0.23 & 0.43 & 0.05 & 0.67 & 0.63 & 0.79 & 0.77 & 0.05 \\
2 & 0.29 & 0.58 & 0.05 & 0.72 & 0.89 & 0.05 & 1.00 & 1.00 & 1.00 & 1.00 & 0.05 \\
3 & 0.11 & 0.27 & 0.07 & 0.26 & 0.46 & 0.08 & 0.71 & 0.67 & 0.81 & 0.79 & 0.10 \\
4 & 0.11 & 0.29 & 0.09 & 0.28 & 0.49 & 0.12 & 0.75 & 0.72 & 0.84 & 0.82 & 0.17 \\
5 & 0.20 & 0.41 & 0.29 & 0.50 & 0.68 & 0.44 & 0.94 & 0.93 & 0.96 & 0.96 & 0.74 \\
6 & 0.26 & 0.45 & 0.36 & 0.62 & 0.75 & 0.55 & 0.98 & 0.98 & 0.99 & 0.98 & 0.87 \\
7 & 0.05 & 0.06 & 0.08 & 0.05 & 0.07 & 0.08 & 0.08 & 0.06 & 0.09 & 0.07 & 0.10 \\
8 & 0.05 & 0.07 & 0.10 & 0.06 & 0.08 & 0.12 & 0.11 & 0.09 & 0.14 & 0.12 & 0.17 \\
9 & 0.09 & 0.18 & 0.29 & 0.21 & 0.33 & 0.46 & 0.61 & 0.57 & 0.68 & 0.65 & 0.74 \\
10 & 0.16 & 0.26 & 0.37 & 0.43 & 0.52 & 0.56 & 0.91 & 0.89 & 0.92 & 0.92 & 0.87 \\
\hline
\end{tabular}

(*) LR and LRE: are the likelihood-ratio using chi-square approximation and the exact distributions; SC and SE are the adapted Sullivan et al. using chi-square approximation and the exact distributions; VMAX test using the exact distribution.

Table 4 - Type I error and power estimates of the tests $-p=2, n=50$ and 100.

\begin{tabular}{|c|c|c|c|c|c|c|c|c|c|c|}
\hline \multicolumn{1}{|c|}{ Average proportion of rejection of the null hypothesis } \\
\hline \multicolumn{1}{|c|}{} & \multicolumn{9}{|c|}{$n=50$} & \multicolumn{5}{|c|}{$n=100$} \\
\hline Case & LR & LRE & SC & SE & VMAX & LR & LRE & SC & SE & VMAX \\
\hline null & 0.06 & 0.05 & 0.06 & 0.05 & 0.05 & 0.05 & 0.05 & 0.05 & 0.05 & 0.05 \\
1 & 0.93 & 0.93 & 0.96 & 0.96 & 0.05 & 1.00 & 0.93 & 1.00 & 1.00 & 0.05 \\
2 & 1.00 & 1.00 & 1.00 & 1.00 & 0.05 & 1.00 & 1.00 & 1.00 & 1.00 & 0.05 \\
3 & 0.95 & 0.95 & 0.97 & 0.97 & 0.13 & 1.00 & 0.95 & 1.00 & 1.00 & 0.18 \\
4 & 0.96 & 0.96 & 0.98 & 0.98 & 0.25 & 1.00 & 0.96 & 1.00 & 1.00 & 0.41 \\
5 & 1.00 & 1.00 & 1.00 & 1.00 & 0.94 & 1.00 & 1.00 & 1.00 & 1.00 & 1.00 \\
6 & 1.00 & 1.00 & 1.00 & 1.00 & 0.99 & 1.00 & 1.00 & 1.00 & 1.00 & 1.00 \\
7 & 0.08 & 0.07 & 0.10 & 0.09 & 0.13 & 0.11 & 0.11 & 0.13 & 0.13 & 0.18 \\
8 & 0.16 & 0.14 & 0.19 & 0.18 & 0.26 & 0.28 & 0.27 & 0.31 & 0.31 & 0.40 \\
9 & 0.89 & 0.88 & 0.92 & 0.91 & 0.94 & 0.99 & 0.99 & 0.99 & 0.99 & 0.99 \\
10 & 0.99 & 0.99 & 0.99 & 0.99 & 0.99 & 1.00 & 1.00 & 1.00 & 1.00 & 1.00 \\
\hline
\end{tabular}

(*) LR and LRE are the likelihood-ratio tests using chi-square and the exact distributions. SC and SE are the adapted Sullivan et al. test using chi-square and the exact distributions. VMAX is the test using the exact distribution. 
values (around 1). On the other hand, the performance of VMAX increased but the SE was still very competitive.

Tables 5 and 6 present the estimates of type I error and power for $p=3$. Considering only the exact distributions to compare the tests (they all reach 0.05 type I error under the null hypothesis), it can be seen that the VMAX did not perform well for cases 2, 3 and 5 (estimated power 0.05 for all sample sizes). These results are expected since the test statistic of VMAX just takes into account changes in variances of the variables. For these models the adapted Sullivan et al. was more efficient than the maximum likelihood ratio test (SE: power $\geq 0.30$; LRE power $\geq 0.16$, for $n \geq 10$ ), particularly for case 5 (power $=0.38$ for $n=5$ and $\geq 0.70$ for $n \geq 10$ ). That was due to the fact that $\boldsymbol{\Sigma}_{\mathbf{1}}$ and $\boldsymbol{\Sigma}_{\mathbf{0}}$ have equal diagonal values although completely different covariance structures.

Table 5 - Type I error and power estimates of the tests $-p=3, n=5,10$ and 25.

\begin{tabular}{|c|c|c|c|c|c|c|c|c|c|}
\hline \multicolumn{8}{|c|}{ Average proportion of rejection of the null hypothesis } \\
\hline & \multicolumn{3}{|c|}{$n=5$} & \multicolumn{3}{|c|}{$n=10$} & \multicolumn{3}{|c|}{$n=25$} \\
\hline Case & LRE & SE & VMAX & LRE & SE & VMAX & LRE & SE & VMAX \\
\hline null & 0.05 & 0.05 & 0.05 & 0.05 & 0.05 & 0.05 & 0.05 & 0.05 & 0.05 \\
1 & 0.31 & 0.73 & 0.24 & 0.88 & 0.98 & 0.40 & 1.00 & 1.00 & 0.70 \\
2 & 0.07 & 0.12 & 0.05 & 0.16 & 0.30 & 0.05 & 0.49 & 0.65 & 0.05 \\
3 & 0.11 & 0.22 & 0.05 & 0.34 & 0.57 & 0.05 & 0.84 & 0.93 & 0.05 \\
4 & 0.05 & 0.06 & 0.11 & 0.05 & 0.07 & 0.15 & 0.08 & 0.11 & 0.21 \\
5 & 0.12 & 0.38 & 0.05 & 0.41 & 0.70 & 0.05 & 0.91 & 0.97 & 0.05 \\
6 & 0.17 & 0.43 & 0.14 & 0.57 & 0.78 & 0.18 & 0.97 & 0.99 & 0.26 \\
7 & 0.20 & 0.46 & 0.17 & 0.63 & 0.82 & 0.25 & 0.99 & 1.00 & 0.40 \\
8 & 0.59 & 0.69 & 0.66 & 0.95 & 0.97 & 0.88 & 1.00 & 1.00 & 1.00 \\
\hline
\end{tabular}

(*) LR and LRE are the likelihood-ratio tests using chi-squared and the exact distributions. SC and SE are the adapted Sullivan et al. test using chi-squared and the exact distributions. VMAX is the test using the exact distribution.

The performance of VMAX improved for the cases which the variances changed as well as the correlation structure (cases 1, 6 and 7), particularly for case 1 whose $\boldsymbol{\Sigma}_{\mathbf{1}}$ is a diagonal matrix and the variance of the first variable doubled the respective value giving in $\boldsymbol{\Sigma}_{\mathbf{0}}$. The adapted Sullivan et al. and the maximum likelihood ratio were still more efficient than VMAX in all cases (power: $\mathrm{SE} \geq 0.78$; LRE: $\geq 0.57$; VMAX: $\geq 0.18$, for $n \geq 10$ ). For larger sample sizes $(n=100)$, the VMAX estimated power for case 1 was equal to SE and LRE values and for $n=50$ they were similar ( 0.93 for VMAX and 1 for the other two tests). In case 6 which corresponds to 1.2 increase of variance, VMAX achieved its maximum estimated power when $n=100$ (power $=0.55$ ), as long as LRE and SE reached power values close to 1 when $n=25$. SE test was the most efficient to detect the increase of variance 1.5 (case 7) since the power estimates are larger or equal 0.82 for $n \geq 10$. Both LRE and SE reached power values closer or 
Table 6 - Type I error and power estimates of the tests $-p=3, n=50$ and 100.

\begin{tabular}{|c|c|c|c|c|c|c|c|c|c|c|}
\hline \multicolumn{1}{|c|}{ Average proportion of rejection of the null hypothesis } \\
\hline & \multicolumn{9}{|c|}{$n=50$} & \multicolumn{5}{|c|}{$n=100$} \\
\hline Case & LR & LRE & SC & SE & VMAX & LR & LRE & SC & SE & VMAX \\
\hline null & 0.06 & 0.05 & 0.07 & 0.05 & 0.05 & 0.06 & 0.05 & 0.05 & 0.05 & 0.05 \\
1 & 1.00 & 1.00 & 1.00 & 1.00 & 0.93 & 1.00 & 1.00 & 1.00 & 1.00 & 1.00 \\
2 & 0.86 & 0.84 & 0.93 & 0.92 & 0.05 & 1.00 & 1.00 & 1.00 & 1.00 & 0.05 \\
3 & 0.99 & 0.99 & 1.00 & 1.00 & 0.05 & 1.00 & 1.00 & 1.00 & 1.00 & 0.05 \\
4 & 0.17 & 0.14 & 0.24 & 0.20 & 0.30 & 0.33 & 0.31 & 0.40 & 0.37 & 0.46 \\
5 & 1.00 & 1.00 & 1.00 & 1.00 & 0.06 & 1.00 & 1.00 & 1.00 & 1.00 & 0.06 \\
6 & 1.00 & 1.00 & 1.00 & 1.00 & 0.37 & 1.00 & 1.00 & 1.00 & 1.00 & 0.55 \\
7 & 1.00 & 1.00 & 1.00 & 1.00 & 0.61 & 1.00 & 1.00 & 1.00 & 1.00 & 0.86 \\
8 & 1.00 & 1.00 & 1.00 & 1.00 & 1.00 & 1.00 & 1.00 & 1.00 & 1.00 & 1.00 \\
\hline
\end{tabular}

(*) LR and LRE are the likelihood-ratio tests using chi-squared and the exact distributions. SC and SE are the adapted Sullivan et al. test using chi-squared and the exact distributions. VMAX is the test using the exact distribution.

equal 1.0 for $n \geq 25$. The best values for VMAX occurred for $n=50$ (power $=0.61$ ) and for $n=100$ (power $=0.86)$.

Although $n=5$ is a small sample size to test hypothesis about the covariance matrix when $p=3$, the adapted Sullivan et al. performed well for the cases 1, 6 and 7, (power: 0.73, 0.43 and 0.46 , respectively), being the estimated power about 3 times larger than VMAX and 2.5 larger than LRE values.

The efficiency of VMAX in detecting the shifts of the covariance matrix improved a lot for case 8 (power: $\geq 0.66 ;=1.0$ for $n \geq 25$ ), case which the covariance structure remains the same as $H_{0}$ but the variance of the second variable (see $\boldsymbol{\Sigma}_{\mathbf{1}}$ ) is 4 times larger than the value giving in $\boldsymbol{\Sigma}_{\mathbf{0}}$. For this particular case, VMAX power estimates were closer to the SE and LRE values.

Comparing the results of cases 4 and 6 (both have $H V R=1.2$, see Table 2), it is possible to see that the different correlation structure affected the SE and LRE efficiencies since the tests were more powerful to detect the shits in case 6 than in the case 4 . This result is due to the fact that the values of SE and LRE test statistics take into account all the variances and covariances values from the $\boldsymbol{\Sigma}_{\mathbf{0}}$ and $\boldsymbol{\Sigma}_{\mathbf{1}}$ matrices, and the matrix $\boldsymbol{\Sigma}_{\mathbf{1}}$ of case 4 is more similar to $\boldsymbol{\Sigma}_{\mathbf{0}}$ than the covariance matrix of case 6 . Both SE and LRE did not perform well in case 4 and VMAX had power estimates larger than these two tests (maximum power estimates: VMAX $=0.46$; $\mathrm{SE}=$ 0.37 ; LRE $=0.31$ ). It is important to point out that cases 4 and 8 belong to the framework which VMAX was proposed by Costa \& Machado (2008; 2009) since only increases of variances were allowed being the correlation, or the covariance, structures the same as in $H_{0}$. Therefore, the results suggest that under this framework the performance of VMAX is similar to the performance of the adapted Sullivan et al. test.

As far as the maximum likelihood-ratio test is concerned its performance was inferior than the adapted Sullivan et al. in all cases for $n \leq 25$ and similar in the majority of cases for $n \geq 50$. 
Considering all the results observed for $p=2$ and 3, we can conclude that for the evaluated cases, when the exact distribution was used to build the critical region of the tests, the adapted Sullivan et al. was more efficient than the maximum likelihood-ratio, both were more efficient than VMAX with few exceptions. The exceptions were related to the cases whose variances changed but the correlation (or the covariance) structure under the alternative hypothesis was kept the same as in $H_{0}$, situations that fit well in the framework of VMAX test. One of the main goals in quality control is to detect variances increases and in this context the results showed that VMAX performed well.

\subsection{Discussion of the results from the asymptotic distribution of the test statistic}

As far as the chi-square approximation is concerned, for the bivariate cases and small samples sizes ( $n=5$ and 10) the maximum likelihood-ratio (LR) and the adapted Sullivan et al. (SC) tests resulted in estimates of type I error larger than the pre-specified nominal significance level of 5\% (estimates were 19 and 10\% respectively, for LR; 12 and $8 \%$ for SC). Therefore, their power estimates could not be compared with the results from the exact distributions of the tests and were not presented in Table 3. For samples of sizes $n=25$ and 50 the estimates were more reasonable (around 6 to $7 \%$ ) and equal $5 \%$ for $n=100$. This is perfectly explained by the fact that the chi-square approximation is valid only for larger sample sizes. For $n \geq 25$ the average power estimates from the chi-square distribution are shown in Tables 3 and 4 . The results were similar to the estimates obtained by the exact distributions.

Similar pattern was found for $p=3$. For small samples $(n=5,10)$, the estimates of the type I error based on the chi-square distribution were also much larger than the pre-specified $5 \%$ significance level. For the maximum likelihood ratio test (LR), the estimates were equal $37 \%$ for $n=5,14 \%$ for $n=10,8 \%$ for $n=25$, being the approximation to $5 \%$ achieved only for samples of sizes 50 and 100 (estimates around 5 to 6\%). For the adapted Sullivan et al. test (SC), the estimates were 22, 14 and $9 \%$ for $n=5,10$ and 25, respectively, achieving 5\% only for larger samples (estimates around 7 to $5 \%$ for $n=50$ and 100). For $n \geq 50$ the average power estimates from the chi-square distribution are shown in Tables 5 and 6 for the LR an SC tests and the results were similar to the estimates obtained by the exact distribution.

Therefore, by the results presented for $p=2,3$, it is clear that the chi-square approximation did not work well for small sample sizes being not recommended in these situations. The exact distribution of the test statistics obtained by Monte Carlo simulation should be used for small sample sizes.

\section{EXAMPLES OF APPLICATION}

In this section two examples of application are presented. The first is a general situation and the second is a particularly case of quality control. In all examples the adapted Sullivan et al. test described in Section 2.4 is compared with the maximum likelihood-ratio and VMAX tests. 
Example 1. The transpiration data of 20 healthy females was given on Table 5.1 from Johnson $\&$ Wichern (2002). The variables of interest were: rate of sweat $\left(X_{1}\right)$, quantity of sodium $\left(X_{2}\right)$ and quantity of potassium $\left(X_{3}\right)$. Suppose the researcher wants to test the null hypothesis $H_{0}$ : $\boldsymbol{\Sigma}=\boldsymbol{\Sigma}_{\mathbf{0}}$ against $H_{1}: \Sigma \neq \Sigma_{\mathbf{0}}$, where

$$
\Sigma_{\mathbf{0}}=\left[\begin{array}{ccc}
3 & 11 & 2 \\
11 & 200 & 6 \\
2 & 6 & 3.74
\end{array}\right]
$$

By the data from Johnson \& Wichern (2002), one can find the maximum likelihood estimate of the population covariance matrix as

$$
\hat{\Sigma}=\left[\begin{array}{rrr}
2.735 & 9.509 & -1.718 \\
9.509 & 189.798 & -5.937 \\
-1.718 & -5.937 & 3.447
\end{array}\right]
$$

The parameter vector under the null hypothesis, the respective maximum likelihood vector estimate and the difference between these two vectors are given by

$$
\begin{gathered}
\theta_{0}=\left(\sigma_{1}, \rho_{12}, \sigma_{2}, \rho_{13}, \rho_{23}, \sigma_{3}\right)^{\prime}=(1.732,0.449,14.142,0.597,0.219,1.933)^{\prime} \\
\hat{\theta}=(1.654,0.417,13.776,-0.559,-0.232,1.856)^{\prime} \\
\hat{\delta}=\hat{\theta}-\theta_{0}=(-0.078,-0.032,-0.366,-1.156,-0.451,-0.076)^{\prime} .
\end{gathered}
$$

The results of the three tests discussed in this paper are shown in Table 7. All tests except VMAX, rejected the null hypothesis. From the structure of $\boldsymbol{\Sigma}_{\mathbf{0}}$ and $\hat{\boldsymbol{\Sigma}}$ matrices, it is easy to notice that VMAX was not sensitive to the covariances deviations from these two matrices. Since the ratios between the variances of $\boldsymbol{\Sigma}_{\mathbf{0}}$ and $\hat{\boldsymbol{\Sigma}}$ are close to 1, the result of VMAX is consistent with its theoretical formulation.

Table 7 - Test statistic values and critical limits - Example 1.

\begin{tabular}{|c|c|c|}
\hline Test & Test statistic & Critical limits $-\alpha=0.05$ \\
\hline LR & 38.69 & 12.59 \\
LRE & 38.69 & 14.23 \\
SC & 85.88 & 12.59 \\
SE & 85.86 & 14.16 \\
VMAX & 0.95 & 1.77 \\
\hline
\end{tabular}

Example 2. In Aparisi et al. (1999) an example was given where three quality characteristics were measure in a part (see Fig. 1): the distance between centers, $X_{1}(\mathrm{~cm})$, and the diameters $X_{2}$ $(\mathrm{cm})$ and $X_{3}(\mathrm{~cm})$. According to the authors the vector mean and the covariance matrix when the process was under control were known and given by

$$
\boldsymbol{\mu}_{\mathbf{0}}=(20,7,4)^{\prime} ; \quad \boldsymbol{\Sigma}_{\mathbf{0}}=\left[\begin{array}{ccc}
0.04 & 0.02 & 0.01 \\
0.02 & 0.02 & 0.011 \\
0.01 & 0.011 & 0.01
\end{array}\right] \text {. }
$$



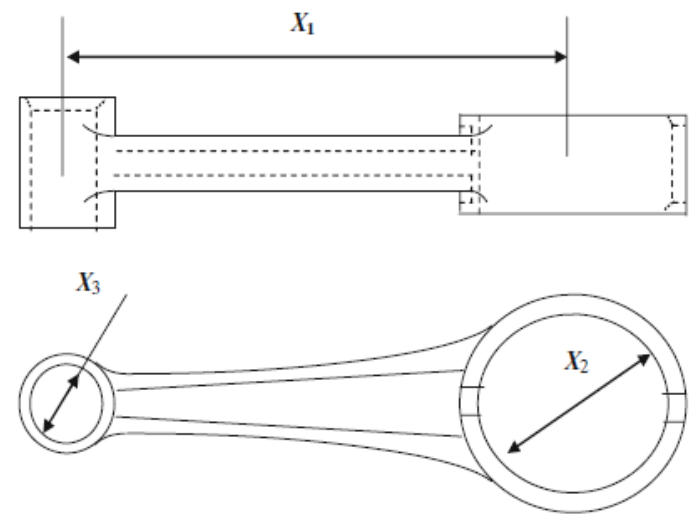

Figure 1 - Part of the example 2. Source: See [Aparisi et. al, 1999].

To illustrate the statistical tests discussed in this paper we perform the following: (i) initially 5 samples of size $n=5,10,25$, were simulated from a multivariate normal distribution with parameters $\boldsymbol{\mu}_{\mathbf{0}}, \boldsymbol{\Sigma}_{\mathbf{0}}$; (2) the remaining 5 samples were generated from a multivariate normal distribution with mean vector $\boldsymbol{\mu}_{\mathbf{0}}$ but different covariance matrices as follows: samples 6 and 7 from matrix $\boldsymbol{\Sigma}_{\mathbf{1}}$, samples 8 and 9 from $\boldsymbol{\Sigma}_{\mathbf{2}}$, sample 10 from $\boldsymbol{\Sigma}_{\mathbf{3}}$, where

$$
\begin{gathered}
\boldsymbol{\Sigma}_{\mathbf{1}}=\left[\begin{array}{rrr}
0.0168 & -0.0053 & 0.0001 \\
-0.0053 & 0.0092 & 0.0002 \\
0.0001 & 0.0002 & 0.0009
\end{array}\right] \boldsymbol{\Sigma}_{\mathbf{2}}=\left[\begin{array}{rrr}
0.0682 & -0.0053 & 0.0001 \\
-0.0053 & 0.0092 & 0.0002 \\
0.0001 & 0.0002 & 0.0009
\end{array}\right] \\
\boldsymbol{\Sigma}_{\mathbf{3}}=\left[\begin{array}{rrr}
0.1288 & -0.0053 & 0.0001 \\
-0.0053 & 0.0092 & 0.0002 \\
0.0001 & 0.0002 & 0.0009
\end{array}\right] .
\end{gathered}
$$

The highest ratio between the variances under the alternative and the null hypothesis were 1.7 for $\boldsymbol{\Sigma}_{\mathbf{2}}$ and 3.2 for $\boldsymbol{\Sigma}_{\mathbf{3}}$. The variances in $\boldsymbol{\Sigma}_{\mathbf{1}}$ are smaller than the variances in $\boldsymbol{\Sigma}_{\mathbf{0}}$. For each sample the null hypothesis $H_{0}: \Sigma=\Sigma_{\mathbf{0}}$, was tested against $H_{1}: \mathbf{\Sigma} \neq \boldsymbol{\Sigma}_{\mathbf{0}}$, by using the maximum likelihoodratio, the adapted Sullivan et al. and VMAX tests at $\alpha=0.005$ significance level. The results are given in Tables 8 and 9 with the critical limits of each test considering the exact distribution as well as the asymptotic chi-square for the maximum likelihood-ratio and the adapted Sullivan et al. tests. For sample sizes of $n=5$, the sample covariance matrices are also shown. By the exact distribution it can be seen that for the first 5 samples the null hypothesis was not rejected by any of the statistical tests and all $n$. For samples $6,7,8$ and $9, H_{0}$ was rejected by the adapted Sullivan et al. (SE) test for all sample sizes; by the maximum likelihood-ratio (LRE) it was rejected for $n=10$ and 25, except for sample 8 whose rejection occurred for $n=25$, only. On the other hand, VMAX rejected the null hypothesis only for sample 9 when $n=25$ and did not rejected for samples 6, 7 and 8 for any sample size.

The null hypothesis was rejected for sample 10 by all statistical tests as expected considering the fact that this sample came from a multivariate normal distribution whose variance of $X_{1}$ is 3.2 times larger than the respective variance given in $\boldsymbol{\Sigma}_{\mathbf{0}}$. 
Table 8 - Results of the Example 2 - samples sizes $n=10$ and $n=25-\alpha=0.005$.

\begin{tabular}{|c|c|c|c|c|c|c|}
\hline \multicolumn{7}{|c|}{ Test Statistics - observed values } \\
\hline & \multicolumn{3}{|c|}{$n=10$} & \multicolumn{3}{|c|}{$n=25$} \\
\hline Sample & LRE & SE & VMAX & LRE & SE & VMAX \\
\hline 1 & 10.4610 & 2.9871 & 1.3467 & 6.6965 & 4.5624 & 1.0794 \\
\hline 2 & 5.4263 & 4.6919 & 1.3168 & 11.4868 & 13.9057 & 1.8518 \\
\hline 3 & 5.7642 & 4.9829 & 1.4768 & 2.5405 & 2.5593 & 1.2564 \\
\hline 4 & 4.8761 & 3.8433 & 0.8694 & 2.4840 & 2.8476 & 1.1432 \\
\hline 5 & 3.4707 & 4.1756 & 1.7074 & 4.7600 & 5.8345 & 1.7261 \\
\hline 6 & 37.6804 & 355.8093 & 0.7003 & 62.1800 & 504.5504 & 0.4022 \\
\hline 7 & 36.0395 & 185.7810 & 0.4293 & 77.2557 & 367.9003 & 0.5291 \\
\hline 8 & 21.1632 & 84.7545 & 1.0847 & 89.3189 & 257.7654 & 1.4477 \\
\hline 9 & 57.8377 & 186.1254 & 2.5899 & 219.0934 & 480.5102 & 4.0263 \\
\hline \multirow[t]{2}{*}{10} & 46.2343 & 60.5017 & 4.2255 & 160.5388 & 207.9995 & 3.4506 \\
\hline & \multicolumn{3}{|c|}{$\begin{array}{c}\mathrm{LLR}=\mathrm{LSC}=18.5476 ; \mathrm{LRE}=24.1789 \\
\mathrm{LSE}=52.0888 ; \mathrm{LVMAX}=2.7559\end{array}$} & \multicolumn{3}{|c|}{$\begin{array}{c}\mathrm{LLR}=\mathrm{LSC}=18.5476 ; \mathrm{LRE}=20.5275 \\
\mathrm{LSE}=29.5167 ; \mathrm{LVMAX}=2.0007\end{array}$} \\
\hline
\end{tabular}

(*) LLR and LSC: likelihood-ratio and the adapted Sullivan et. al tests critical limits using chi-square distribution. LRE and LSE: likelihood-ratio and the adapted Sullivan et. al tests critical limits using exact distribution. LVMAX: critical limit for VMAX test.

Considering the asymptotic chi-square distribution the null hypothesis was rejected for samples 6-10 by likelihood-ratio (LR) as well as by the adapted Sullivan et al. (SC) for all sample sizes. However, it is important to remind that as shown in Section 3.2, for $p=3$ and $n=5,10,25$, the type I error of these two tests for small samples is inflated being larger than the pre-specified significance level, particularly for $n=5,10$.

It is interesting to point out that the same parameters $\boldsymbol{\mu}_{\mathbf{0}}$ and $\boldsymbol{\Sigma}_{\mathbf{0}}$ were used by Costa \& Machado (2009), in the illustrative example given on Section 5 of their paper. The matrix $\boldsymbol{\Sigma}_{\mathbf{1}}$, which was used in our example, is the covariance matrix of the sample number 4 presented in Costa \& Machado's example (2009), and it was considered similar to the matrix $\boldsymbol{\Sigma}_{\mathbf{0}}$ by the VMAX test in their paper.

\section{FINAL REMARKS}

Considering the exact distribution of the tests statistics, in the majority of the simulated cases, the adapted Sullivan et al. and the likelihood-ratio tests resulted in larger values of estimated power than VMAX for bivariate and trivariate normal distributions. VMAX was not sensitive to general changes in the covariance (or correlation) structure. Its estimated powers increased when the variances were larger under the alternative than the null hypothesis and the covariance (or correlation) structure remained similar under $H_{0}$ and $H_{1}$. This result is no very surprising since VMAX was built with the main purpose of detecting variances shifts and it has been proved to be efficient on this type of situation (see Costa \& Machado, 2008, 2009; Quinino et al., 2012). It still can be used for this purpose but it is important to point out that the adapted Sullivan et al. was also competitive in these particular cases. 
Table 9 - Results of the Example 2 - sample size $n=5$.

\begin{tabular}{|c|c|c|c|c|c|c|c|}
\hline & & amplo сочи & rians matri & & Test & tatistic $-\mathrm{O}$ & d values \\
\hline & & 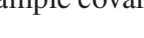 & mance matri & & $\overline{L R E}$ & SE & VMAX \\
\hline & & 0.0970 & 0.0419 & 0.0203 & & & \\
\hline 1 & $S_{1}=$ & 0.0419 & 0.0245 & 0.0123 & 5.8330 & 5.2599 & 1.9393 \\
\hline & & 0.0203 & 0.0123 & 0.0073 & & & \\
\hline & & 0.0189 & 0.0012 & 0.0044 & & & \\
\hline 2 & $S_{2}=$ & 0.0012 & 0.0077 & 0.0069 & 5.4071 & 12.8939 & 1.0760 \\
\hline & & 0.0044 & 0.0069 & 0.0119 & & & \\
\hline & & 0.0265 & 0.0176 & 0.0144 & & & \\
\hline 3 & $S_{3}=$ & 0.0176 & 0.0363 & 0.0158 & 11.4558 & 10.3729 & 1.5729 \\
\hline & & 0.0144 & 0.0158 & 0.0102 & & & \\
\hline & & 0.0238 & 0.0007 & 0.0010 & & & \\
\hline 4 & $S_{4}=$ & 0.0007 & 0.0079 & 0.0069 & 4.5389 & 10.4866 & 0.7665 \\
\hline & & 0.0010 & 0.0069 & 0.0096 & & & \\
\hline & & 0.0183 & 0.0128 & 0.0050 & & & \\
\hline 5 & $S_{5}=$ & 0.0128 & 0.0128 & 0.0049 & 12.0149 & 8.5493 & 0.7537 \\
\hline & & 0.0050 & 0.0049 & 0.0024 & & & \\
\hline & & 0.0131 & -0.0055 & 0.0019 & & & \\
\hline 6 & $S_{6}=$ & -0.0055 & 0.0067 & -0.0004 & 23.6423 & 201.5283 & 0.2886 \\
\hline & & 0.0019 & -0.0004 & 0.0006 & & & \\
\hline & & 0.0184 & -0.0086 & 0.0015 & & & \\
\hline 7 & $S_{7}=$ & -0.0086 & 0.0092 & -0.0001 & 19.4767 & 155.7951 & 0.4992 \\
\hline & & 0.0015 & -0.0001 & 0.0010 & & & \\
\hline & & 0.0322 & -0.0126 & 0.0027 & & & \\
\hline 8 & $S_{8}=$ & -0.0126 & 0.0117 & 0.0000 & 20.3896 & 158.6873 & 1.2901 \\
\hline & & 0.0027 & 0.0000 & 0.0016 & & & \\
\hline & & 0.0850 & 0.0039 & 0.0047 & & & \\
\hline 9 & $S_{9}=$ & 0.0039 & 0.0223 & -0.0032 & 33.6708 & 215.0552 & 3.0146 \\
\hline & & 0.0047 & -0.0032 & 0.0010 & & & \\
\hline & & 0.3802 & -0.0113 & -0.0047 & & & \\
\hline 10 & $S_{10}=$ & -0.0113 & 0.0175 & 0.0008 & 79.7073 & 131.9005 & 7.6054 \\
\hline & & -0.0047 & 0.0008 & 0.0006 & & & \\
\hline
\end{tabular}

(*) LLR and LSC: likelihood-ratio and the adapted Sullivan et al. tests critical limits using chi-square distribution. LRE and LSE: likelihood-ratio and the adapted Sullivan et. al tests critical limits using exact distribution. LVMAX: critical limit for VMAX test.

As far as the efficiency of the likelihood-ratio test is concerned, the results showed that the adapted Sullivan et al. test was more efficient in most of the cases, except for larger samples were their performance were similar. In fact for $p=3$ the adapted Sullivan et al. was always superior. In some cases for $p=2$, VMAX was more efficient than the likelihood-ratio test. 
The results presented in this paper had indicated that the adapted Sullivan et al. is a good alternative for testing hypothesis about the structure of the covariance matrix. It is a very flexible test and it can be applied in more general situations to detect differences in the variances as well as in other parameters of the covariance matrix. Also the fact that the adapted Sullivan et al. test depends upon the calculation of Fisher Information matrix, makes it possible to be used for multivariate normal and non-normal populations.

For small samples sizes the chi-square approximation did not performed well being not recommended since the estimates of type I error were larger than the pre-specified significance level for the adapted Sullivan et al. and for the likelihood-ratio tests. However, this fact does not make the use of the adapted Sullivan et al. or the likelihood-ratio tests restrictive since the exact distribution of the respective test statistics under the null hypothesis are easily obtained by Monte Carlo simulation. The same is true for the VMAX test for $p>2$ since in these situations the use of numerical integration to find the critical region of the test is more complex and Monte Carlo simulation can be very helpful.

\section{ACKNOWLEDGMENTS}

This work was partially supported by the Brazilian Institutions CNPq and CAPES (PROCADProject). The authors wish to thank the anonymous referees for the suggestions that led to the improvement of the paper.

\section{REFERENCES}

[1] Alt F. 1985. Multivariate quality control. In: Encyclopedia of Statistical Sciences [edited by S. Kotz, \& N.L. Johnson], vol. 6, John Wiley, 110-122.

[2] Anderson TW. 1958. An Introduction to Multivariate Statistical Analysis. New York: John Wiley \& Sons, Inc.

[3] Aparisi F, Jabaioyes J \& CARrion A. 1999. Statistical properties of the $|s|$ multivariate control chart. Communications in Statistics-Theory and Methods, 28(11): 2671-2686.

[4] Beyer WH. 1978. Standard Mathematical Tables. West Palm Beach: CRC.

[5] Casella G \& Berger RL. 2002. Statistical Inference. California: Duxbury Thomson Learning.

[6] Costa AFB \& Machado MAG. 2009. A new chart based on sample variances for monitoring the covariance matrix of multivariate processes. International Journal of Advanced Manufacturing Technology, 41(7-8): 770-779.

[7] Costa AFB \& MACHAdo MAG. 2008. A new multivariate chart for monitoring the covariance matrix of bivariate processes. Communications in Statistics. Simulation and Computation, 37(7): 14531465 .

[8] Davis PJ \& Rabinowitz P. 1984. Methods of Numerical Integration. Orlando: Academic Press.

[9] DJauhari MA. 2009. Asymptotic distribution of sample covariance determinant. Matematika, 25(1): 79-85. 
[10] DJAUHARI MA. 2005. Improved monitoring of multivariate process variability. Journal of Quality Technology, 37(1): 32-39.

[11] GARCíA-DiAz JC. 2007. The effective variance control chart for monitoring the dispersion process with missing data. European Journal of Industrial Engineering, 1(1): 40-55.

[12] Johnson RA \& Wichern WD. 2002. Applied Multivariate Statistical Analysis. New Jersey: Prentice Hall.

[13] LI Y \& TSUNG F. 2011. Detecting and diagnosing covariance matrix changes in multistage processes. IIE Transactions, 43(4): 259-274.x

[14] Quinino R, Costa A \& Ho LL. 2012. A single statistic for monitoring the covariance matrix of bivariate processes. Quality Engineering, 24(3): 423-430.

[15] Montgomery DC. 2008. Introduction to Statistical Quality Control. New York: John Wiley.

[16] Sullivan JH, Stoumbos ZG, Mason RL \& Young JC. 2007. Step-down analysis for change in the covariance matrix and other parameters. Journal of Quality Technology, 39(1): 66-84.

[17] YeH AB, Li B \& WANG K. 2012. Monitoring multivariate process variability with individual observations via penalised likelihood estimation. International Journal of Production Research, 50(22): 6624-6638.

[18] Yeh AB, Lin DKJ \& MCGRATh RN. 2006. Multivariate control charts for monitoring covariance matrix: a review. Quality of Technology and Quantitative Managament, 3(4): 415-436. 